\title{
ChREBP Rather Than SHP Regulates Hepatic VLDL Secretion
}

\author{
Hiroyuki Niwa ${ }^{1}$ (D) , Katsumi Iizuka ${ }^{1,2, *}$ (i) , Takehiro Kato ${ }^{1}$, Wudelehu $\mathrm{Wu}^{1}{ }^{1}$, Hiromi Tsuchida ${ }^{1}$, \\ Ken Takao ${ }^{1}$, Yukio Horikawa ${ }^{1}$ and Jun Takeda ${ }^{1}$ \\ 1 Department of Diabetes and Endocrinology, Graduate School of Medicine, Gifu University, Gifu 501-1194, \\ Japan; hiroyu760202@yahoo.co.jp (H.N.); bado_aberu@yahoo.co.jp (T.K.); wudelehu100@126.com (W.W.); \\ Gif095@gifu-u.ac.jp (H.T.); lamgerrpard@yahoo.co.jp (K.T.); yhorikaw@gifu-u.ac.jp (Y.H.); \\ jtakeda@gifu-u.ac.jp (J.T.) \\ 2 Gifu University Hospital Center for Nutritional Support and Infection Control, Gifu 501-1194, Japan \\ * Correspondence: kiizuka@gifu-u.ac.jp; Tel.: +81-58-230-6564; Fax: +81-58-230-6376
}

Received: 6 February 2018; Accepted: 5 March 2018; Published: 7 March 2018

\begin{abstract}
The regulation of hepatic very-low-density lipoprotein (VLDL) secretion plays an important role in the pathogenesis of dyslipidemia and fatty liver diseases. VLDL is controlled by hepatic microsomal triglyceride transfer protein (MTTP). Mttp is regulated by carbohydrate response element binding protein (ChREBP) and small heterodimer partner (SHP). However, it is unclear whether both coordinately regulate Mttp expression and VLDL secretion. Here, adenoviral overexpression of ChREBP and SHP in rat primary hepatocytes induced and suppressed Mttp mRNA, respectively. However, Mttp induction by ChREBP was much more potent than suppression by SHP. Promoter assays of Mttp and the liver type pyruvate kinase gene revealed that SHP and ChREBP did not affect the transcriptional activity of each other. Mttp mRNA and protein levels of Shp ${ }^{-/-}$mice were similar to those of wild-types; however, those of $\mathrm{Chrebp}^{-/-} \mathrm{Shp}^{-/-}$and Chrebp ${ }^{-/-}$mice were significantly much lower. Consistent with this, the VLDL particle number and VLDL secretion rates in $\mathrm{Shp}^{-/-}$mice were similar to wild-types but were much lower in Chrebp ${ }^{-/-}$and $\mathrm{Chrebp}^{-/-} \mathrm{Shp}^{-/-}$ mice. These findings suggest that ChREBP, rather than SHP, regulates VLDL secretion under normal conditions and that ChREBP and SHP do not affect the transcriptional activities of each other.
\end{abstract}

Keywords: carbohydrate response element binding protein; small heterodimer partner; microsomal triglyceride transfer protein; very-low-density lipoprotein

\section{Introduction}

Nonalcoholic fatty liver disease (NAFLD) is the most common chronic liver disease in the world [1,2]. High fat diets and high carbohydrate diets are responsible in the development of NAFLD [3-5]. High carbohydrate diets cause fatty liver by increasing hepatic de novo lipogenesis [3]. High fat diets increase hepatic fat content [4]. Therefore, lifestyle modifications, including hypocaloric diets, are critical for the prevention and treatment of NAFLD. Non-alcoholic fatty liver disease (NAFLD) is associated with hepatic insulin resistance and hepatic fibrosis and can lead to the development of diabetes mellitus, dyslipidemia, non-alcoholic steatohepatitis, and hepatocellular carcinoma $[1,2]$. Hepatic lipid accumulation is regulated by the free fatty acid (FFA) supply from adipose tissue, and de novo lipogenesis from the secretion of glucose, acyl CoA oxidation, and very-low-density lipoprotein (VLDL) in the liver [6]. Visceral adipose tissue is the primary source of hepatic fat in adults, contributing 59\% of the triglycerides found in the liver [6]. Glucose and insulin signals regulate hepatic de novo lipogenesis through the carbohydrate response element binding protein (ChREBP) and sterol response element binding protein 1c (SREBP1c), respectively [7]. Moreover, altered VLDL secretion also contributes to the pathogenesis of NAFLD [8,9]. VLDL secretion is 
controlled by microsomal triglyceride transfer protein (MTTP) [8,9]. MTTP is essential for the assembly and secretion of apolipoprotein B-containing lipoproteins. In humans and mice, Mttp deficiency causes hypolipidemia and fatty liver [8,9]. Thus, Mttp is involved in the pathogenesis of NAFLD.

Mttp regulation depends on a few highly-conserved cis-elements in its promoter. The Mttp promoter sequence contains critical positive (hepatic nuclear factor [HNF]-1, HNF-4, direct repeat 1 , and FOX) and negative regulatory sterol and insulin response elements [8,9]. Small heterodimer partner (SHP, also known as NR0B2) is a unique nuclear receptor (NR) that contains the dimerization and ligand-binding domain found in other family members but lacks the conserved DNA-binding domain [10]. We previously reported that human SHP genetic variations appear to cause mild obesity and type 2 diabetes mellitus [11,12]. As a co-repressor, SHP represses the activities of HNF- $4 \alpha$ and the retinoid $X$ receptor liver receptor homolog-1 (LRH-1) by interacting with these factors $[13,14]$. However, it is not certain whether SHP affects the VLDL secretion rate [15,16].

As with the effects of nutritional conditions on Mttp expression, high fat diets and high carbohydrate diets induce Mttp expression, resulting in hyperlipidemia $[17,18]$. High fat diets increase hepatic Mttp expression through decreased binding of sterol regulatory element-binding proteins to the $M t t p$ promoter [17]. High sucrose and high fructose consumption also increase Mttp expression [18]; however, the mechanisms of this are unclear. Recently, we reported that Mttp mRNA levels and VLDL secretion rates were lower in the livers of Carbohydrate Response Element Binding Protein [ChREBP] knockout $\left(\mathrm{Chrebp}^{-/-}\right)$mice [19]. ChREBP is a glucose-activated transcription factor that regulates hepatic de novo lipogenesis in the liver [20-23]. ChREBP transcriptional activities are regulated by phosphorylation/dephosphorylation, nuclear translocation, and conformational changes [20-23]. It was recently proposed that ChREBP transcriptional activity is regulated through interactions with nuclear factors, such as farnesoid X receptor (FXR) and HNF4a [24-28]. Moreover, computer analysis revealed that ChREBP contains a nuclear receptor binding motif [29]. Considering that SHP represses the activities of many transcription factors by interacting with these factors [10], we speculated that SHP might also affect $M t t p$ transcription in cooperation with ChREBP.

ChREBP and SHP control the regulation of Mttp expression. Using rat hepatocytes and knockout mice, we therefore evaluated the following: (1) whether ChREBP and SHP affect the transcriptional activities of each other; (2) whether ChREBP and SHP coordinately affect Mttp expression and thereby VLDL secretion; and (3) if ChREBP or SHP regulate VLDL secretion more potently. An appreciation of the roles of ChREBP and SHP in regulating Mttp and VLDL secretion will be beneficial for understanding the role of nutritional signals in the development of NAFLD and dyslipidemia.

\section{Materials and Methods}

\subsection{Establishment of Chrebp ${ }^{-/-} \mathrm{Shp}^{-/-}$Double Knockout (DKO) Mice}

Animal experiments were carried out in accordance with the National Institute of Health Guide for the Care and Use of Laboratory Animals (NIH Publications No. 8023, revised 1978). All animal care was approved by the animal care committee of the University of Gifu (No. 27-30, approval date: 4 June 2015). Mice were housed at $23^{\circ} \mathrm{C}$ on a 12-h light/dark cycle. Chrebp ${ }^{-/-}$mice were backcrossed for at least 10 generations onto the C57BL/6J background [19,30]. Shp ${ }^{-/-}$mice were purchased from Lexicon Genetics Inc. (The Woodlands, TX, USA). Shp ${ }^{+/-}$mice were backcrossed for at least 12 generations onto the C57BL/6J background. Male mice were used for all studies. Chrebp ${ }^{-/-} \mathrm{Shp}^{-/-}$ (DKO) mice were intercrossed with Chrebp ${ }^{-/-}$and Shp ${ }^{-1-}$ mice.

Mice had free access to water and were fed an autoclaved CE-2 diet (CLEA Japan, Tokyo, Japan). Wild-type (WT), Chrebp ${ }^{-/-}, \mathrm{Shp}^{-/-}$, and DKO mice were housed separately with a total of three mice per cage. Body weight was measured weekly between 7 and 21 weeks of age. Mice were sacrificed at 21 weeks of age by cervical dislocation. All tissue samples were immediately placed into liquid nitrogen and stored at $-80^{\circ} \mathrm{C}$ until further analysis for hepatic triacylglycerol and cholesterol contents and for quantitative polymerase chain reaction (PCR). 


\subsection{Liver Triglyceride and Cholesterol Content and Plasma Profile Measurements}

Liver lipids were extracted using the Bligh and Dyer method [31]; they were measured using triglyceride (Wako Pure Chemicals, Osaka, Japan) and cholesterol E-tests (Wako). Blood plasma was collected from the retro-orbital venous plexus, following ad libitum feeding or after a 6-h fast. Blood glucose and beta-hydroxybutyrate $(\beta-\mathrm{OHB})$ levels were measured using a FreeStyle Freedom monitoring system (Nipro, Osaka, Japan). Plasma insulin, FFA, fibroblast growth factor 21 (FGF21), triglyceride, and total cholesterol levels were determined using commercial assay kits, as follows: mouse insulin enzyme-linked immunosorbent assay (ELISA) (H type) (Shibayagi, Gunma, Japan), NEFA C-test (Wako Pure Chemicals, Tokyo, Japan), mouse/rat Fgf21 ELISA (R\&D Systems, Minneapolis, MN, USA), triglyceride E-test (Wako), and the cholesterol E-test (Wako), respectively.

\subsection{RNA Isolation and Quantitative Real-Time PCR}

Total RNA isolation, cDNA synthesis, and real-time PCR analysis were performed as previously described [13]. Real-time PCR primers for mouse/rat Chrebp, liver type pyruvate kinase (Pklr), Fgf21, Mttp, and RNA polymerase II (Pol2) have been previously reported [31-33]. All amplifications were performed in triplicate. The relative amounts of mRNA were calculated using the comparative CT method. Pol2 expression was used as an internal control.

\subsection{VLDL Secretion Test and MTTP Protein Contents}

VLDL secretion tests were performed as previously reported [19]. Briefly, $500 \mathrm{mg} / \mathrm{kg}$ body weight tyloxapol was administered intraperitoneally to $5 \mathrm{~h}$ fasted mice. Blood sampling was performed at the indicated times. The triglyceride (TG) content in lipoprotein fractions and the VLDL particle numbers were analyzed using gel-permeation high-performance liquid chromatography (LipoSEARCH ${ }^{\circledR}$ ) at Skylight Biotech Inc. (Akita, Japan) [34]. Serum samples obtained from six mice after $6 \mathrm{~h}$ fasting were pooled and measured. Liver MTTP protein contents were measured by an MTTP ELISA kit (Cloud-Clone Corp Inc., Houston, TX, USA).

\subsection{Adenoviral Delivery into Rat Primary Hepatocytes}

Rat primary hepatocytes were isolated as previously described $[32,33]$. Isolated hepatocytes were suspended in DMEM, supplemented with 10\% fetal calf serum, $100 \mathrm{nM}$ insulin, $100 \mathrm{nM}$ dexamethasone, $10 \mathrm{nM}$ triiodothronine (T3), and $100 \mu \mathrm{g} / \mathrm{mL}$ penicillin/streptomycin. Cells were seeded in 6-well plates or $10-\mathrm{cm}$ dishes and grown in a humidified atmosphere of $5 \% \mathrm{CO}_{2}$ and $95 \%$ air at $37^{\circ} \mathrm{C}$. After the cells had been incubated for $4 \mathrm{~h}$, the medium was replaced with DMEM containing $10 \mathrm{nM}$ T3. Adenoviruses harboring dominant active rat Chrebp lacking 1-196 a.a. (Ad-daChREBP) and mouse SHP full length (Ad-SHP) were constructed according to the manufacturer's protocol (Invitrogen, Carlsbad, CA, USA). After $4 \mathrm{~h}$ infection with Ad-daChREBP and/or Ad-SHP, rat hepatocytes were incubated for 20 h. Mttp mRNA levels were detected by real-time PCR.

\subsection{Transfections and Luciferase Reporter Assay}

Reporter plasmids pGL3 $3 \times$ LPK ChoRE, pcDNA-daChrebp, and pcDNA-empty have been previously reported [32,33]. pcDNA-SHP and pcDNA-FXR were constructed using the pcDNA ${ }^{\text {TM} 3.2-D E S T ~ M a m m a l i a n ~ E x p r e s s i o n ~ V e c t o r ~(I n v i t r o g e n) ~ a c c o r d i n g ~ t o ~ t h e ~ m a n u f a c t u r e r ' s ~}$ protocol (Invitrogen). pGL3-Mttp (-211 bp) was constructed as follows: mouse Mttp promoter regions $(-211$ bp to $+77 \mathrm{bp})$ amplified by PrimSTAR MAX DNA Polymerase (Takara Bio, Kusatsu, Japan) were inserted into the pGL3 basic vector (Promega, Madison, WI, USA). Then, $1.0 \mu \mathrm{g}$ of pGL3 MTTP (-211 bp), $0.1 \mu \mathrm{g}$ pRL-TK vector, pcDNA-empty + pcDNA-daChREBP + pcDNA-SHP (total $1.0 \mu \mathrm{g}$ ), and $3 \mu \mathrm{L}$ of Lipofectamine 2000 (Invitrogen) were transfected into primary rat hepatocytes. After incubation for $24 \mathrm{~h}$, cells were collected and used in luciferase assays. 


\subsection{Statistical Analysis}

All values are presented as means \pm standard deviations. Data were analyzed using Tukey's test. A $p$-value $<0.05$ was considered to be statistically significant.

\section{Results}

\subsection{Adenoviral Overexpression of ChREBP and SHP Respectively Increased and Decreased Mttp Expression}

Adenoviral ChREBP caused an increase in Mttp and Pklr mRNA expression, while SHP suppressed only Mttp expression in primary rat hepatocytes (Figure 1A,B). Moreover, overexpressing SHP by more than 70-fold (above physiological levels) was found to suppress ChREBP-mediated Mttp induction (Figure 1A). In contrast, SHP overexpression failed to suppress ChREBP-mediated Pklr induction (Figure 1B). This suggested that ChREBP induced Mttp expression more potently than SHP suppressed it.

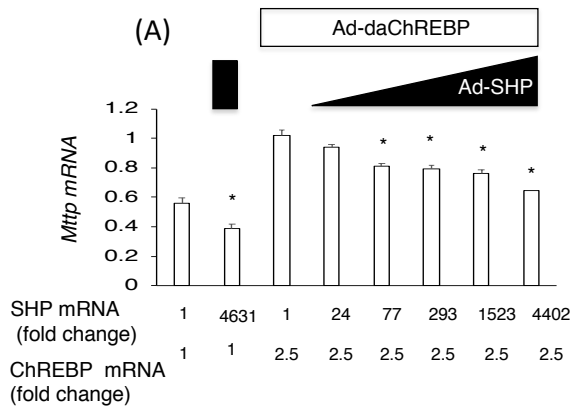

(C)

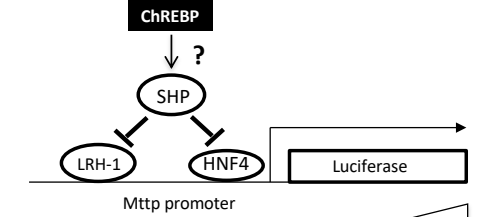

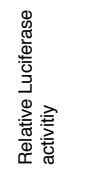

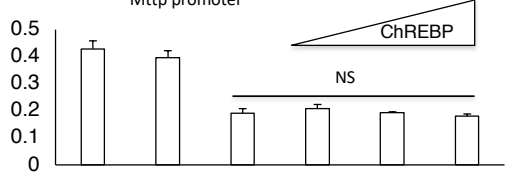

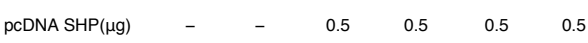
pcDNAPcDNA-
daChREBP $(\mu \mathrm{g})$

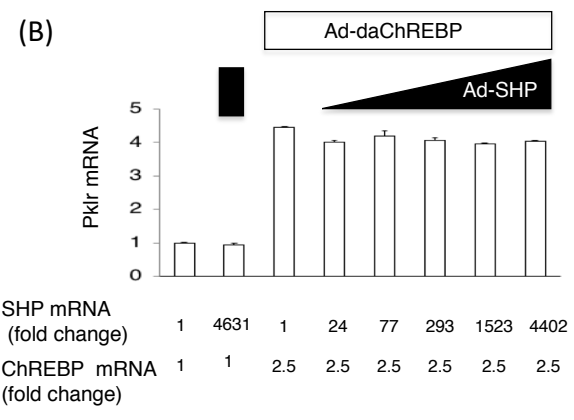

(D)

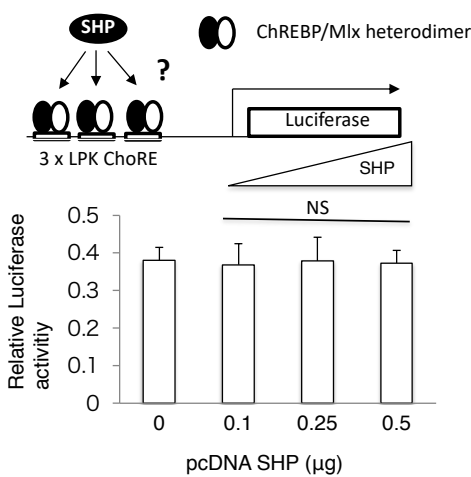

Figure 1. The coordinated effects of carbohydrate response element binding protein (ChREBP) and small heterodimer partner (SHP) on Mttp expression. (A) and (B) The effects of SHP on ChREBP-mediated Mttp (A) and Pklr (B) mRNA induction. After $4 \mathrm{~h}$ infection with Ad-daChREBP and/or Ad-SHP in hepatocytes, cells were incubated for $20 \mathrm{~h}$. Mttp mRNA levels were detected by real-time Polymerase chain reaction method. Open symbols and closed symbols indicated adenoviral overexpression of daChREBP and SHP, respectively. The $x$-axis indicates Shp and Chrebp mRNA levels (fold change). Adenoviral overexpression of ChREBP caused a 2.5-fold increase in Chrebp mRNA levels. Adenoviral SHP expression caused an indicated fold increase in Shp expresssion. Pol2 expression was used as an internal control. $n=4$ per group. ${ }^{*} p<0.05$; (C) Reporter assay using pGL3-Mttp ( $-211 \mathrm{bp}$ ). The indicated amounts of pcDNA-daChREBP and pcDNA-SHP were transfected with pGL3-Mttp ( $-211 \mathrm{bp}$ ) and pRL-TK vectors and Lipofectamine 2000 reagent into primary rat hepatocytes. After $24 \mathrm{~h}$ incubation, cells were collected for the dual luciferase assay. Luciferase activity was normalized to Renilla luciferase activity. N.S., not significant. $n=6$ per group; (D) Reporter assay using pGL3 $3 \times$ LPK ChoRE. The indicated amount of pcDNA-SHP was cotransfected with pGL3-3 $\times$ LPK ChoRE, PRL-TK, pcDNA daChREBP, and Lipofectamine 2000 into primary rat hepatocytes. After $24 \mathrm{~h}$ incubation, cells were collected for the dual luciferase assay. Luciferase activity was normalized to Renilla luciferase activity. NS, not significant vs control. $n=6$ per group. 
SHP is known to suppress HNF4/HNF1/LRH-1-mediated Mttp expression [13,35]. Accordingly, transfection of the PGL3 basic vector containing $211 \mathrm{bp}$ of the Mttp promoter region (pcDNA-SHP), including the HNF4/HNF1/LRH-1 binding site, successfully suppressed PGL3-Mttp (-211 bp) luciferase activity. However, co-transfection of pcDNA-daChREBP did not reverse this effect (Figure 1C). In contrast, the transfection of the pcDNA SHP vector could not suppress pGL3 Pklr luciferase activities induced by pcDNA-daChREBP (Figure 1D). This suggested that SHP and ChREBP did not affect the transcriptional activities of each other

\subsection{Chrebp Shp DKO Mice Resembled Chrebp ${ }^{-/-}$Mice}

We next assessed the effects of ChREBP and SHP on the mouse metabolic phenotype in vivo. Chrebp $^{-/-}$mice displayed characteristically higher liver weights, lower white adipose tissue weights, higher plasma FFA, FGF-21, and $\beta$-OHB levels, and elevated liver glycogen contents compared with those of WT mice. Chrebp ${ }^{-/-} \mathrm{Shp}^{-/-}$double knockout (DKO) mice also displayed similar characteristics (Table 1).

Table 1. Phenotypic comparison between WT, $\mathrm{ChREBP}^{-/-}, \mathrm{SHP}^{-/-}, \mathrm{ChREBP}^{-/-} \mathrm{SHP}^{-/-}$(DKO) mice.

\begin{tabular}{ccccc}
\hline & WT & ChREBP $^{-I-}$ & SHP $^{-I-}$ & DKO \\
\hline BW (g) & $29.50 \pm 0.71$ & $30.40 \pm 1.92$ & $28.57 \pm 2.98$ & $30.2 \pm 2.51$ \\
Liver (\%BW) & $5.02 \pm 0.08$ & $5.37 \pm 0.27^{*}$ & $5.01 \pm 0.14$ & $5.52 \pm 0.24^{*}$ \\
Epidydimal fat (\%BW) & $2.41 \pm 0.30$ & $1.68 \pm 0.62^{*}$ & $2.07 \pm 0.33$ & $1.41 \pm 0.24^{*}$ \\
Mesenteric fat (\%BW) & $1.16 \pm 0.13$ & $0.77 \pm 0.16^{*}$ & $1.02 \pm 0.28$ & $0.55 \pm 0.11^{*}$ \\
Brown adipose tissue (\%BW) & $0.30 \pm 0.07$ & $0.27 \pm 0.10$ & $0.36 \pm 0.12$ & $0.49 \pm 0.15$ \\
Plasma glucose (mg/dL) & $132.3 \pm 17.3$ & $128.4 \pm 13.2$ & $118.7 \pm 21.5$ & $117.2 \pm 11.5$ \\
Plasma insulin (ng/dL) & $0.86 \pm 0.26$ & $1.01 \pm 0.33$ & $0.91 \pm 0.41$ & $0.65 \pm 0.17$ \\
HOMA-R (m) & $0.28 \pm 0.08$ & $0.32 \pm 0.12$ & $0.27 \pm 0.15$ & $0.19 \pm 0.03$ \\
Plasma triglyceride (mg/dL) & $66.1 \pm 10.1$ & $56.2 \pm 11.5$ & $65.2 \pm 12.6$ & $67.3 \pm 19.8$ \\
Plasma FGF21 (pg/mL) & $802.1 \pm 365.8$ & $150.2 \pm 51.1 *$ & $584.1 \pm 268.6$ & $124.0 \pm 61.8^{*}$ \\
Plasma $\beta$-hydroxybutyrate (mM) & $2.05 \pm 0.24$ & $1.32 \pm 0.11$ & $2.37 \pm 0.40$ & $1.49 \pm 0.22$ \\
Liver glycogen content (mg/g liver) & $40.5 \pm 12.4$ & $80.8 \pm 20.2^{*}$ & $33.4 \pm 10.5$ & $78.0 \pm 22.7^{*}$ \\
Liver cholesterol content (mg/g liver) & $8.82 \pm 1.63$ & $9.54 \pm 1.02$ & $8.98 \pm 1.53$ & $7.17 \pm 1.66$ \\
Liver triglyceride content (mg/g liver) & $17.3 \pm 4.2$ & $16.0 \pm 3.77$ & $17.2 \pm 5.14$ & $15.7 \pm 3.17$ \\
\hline
\end{tabular}

BW, body weight; EP, epidydimal fat weight; VS, visceral fat weight; BAT, brown adipose tissue weights; FGF21, fibroblast growth factor $21 .{ }^{*} p<0.05$ vs. WT.

Chrebp and Shp mRNA levels were unaffected by Shp and Chrebp deletions, respectively (Figure 2A,B). Consistent with these phenotypes, the expression levels of ChREBP target genes, such as Pklr and $F g f 21$, were significantly lower in $\mathrm{Chrebp}^{-/-}$and DKO mice (Figure 2C,D). In contrast, the expression of Cyp7a1, a SHP target gene, was significantly higher in Shp ${ }^{-1-}$ and DKO mice but was unchanged in Chrebp ${ }^{-/-}$mice (Figure 2E). Many phenotypes seen in DKO mice were similar to those of $\mathrm{Chrebp}^{-/-}$mice, suggesting that SHP had little effect on ChREBP target gene expression. 
(A)

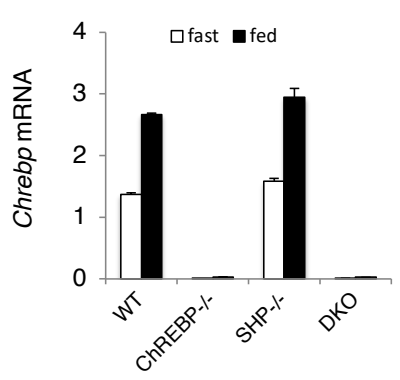

(D)

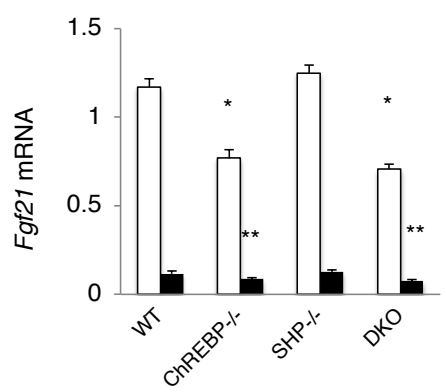

(B)

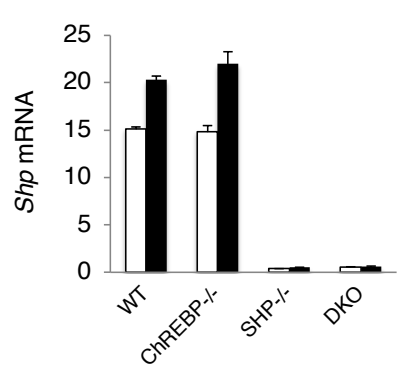

(C)

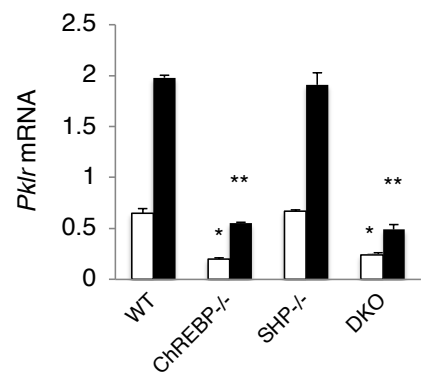

(E)

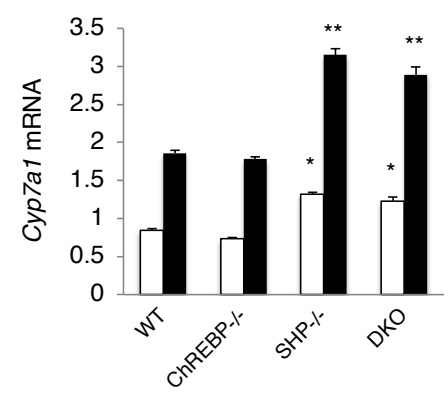

Figure 2. ChREBP and SHP target mRNA levels in wild-type, $\mathrm{Chrebp}^{-/-}, \mathrm{Shp}^{-/-}$, and Chrebp ${ }^{-/-}$ $\mathrm{Shp}^{-1-}$ mice. Carbohydrate response element binding protein (Chrebp) (A); small heterodimer partner (Shp) (B); liver type pyruvate kinase (Pklr) (C); fibroblast growth factor 21 (Fgf21) (D); and Cholesterol 7 alpha-hydroxylase (Cyp7a1); (E) mRNA expression analysis in the livers of wild-type, Chrebp ${ }^{-/-}$, $\mathrm{Shp}^{-1-}$, and $\mathrm{Chrebp}^{-1-} \mathrm{Shp}^{-1-}$ (DKO) mice. Open squares indicate fasted conditions; closed squares indicate fed conditions. Pol2 expression was used as an internal control. Each mRNA levels were corrected with mouse RNA polymerase 2 mRNA. ${ }^{*} p<0.05$ vs. WT (fast), $n=4 .{ }^{* *} p<0.05$ vs. WT (fed), $n=4$.

\subsection{ChREBP and SHP Respectively Positively and Negatively Controlled VLDL Secretion through Mttp Regulation}

We evaluated the effects of Chrebp and Shp deletion on Mttp expression. Hepatic Mttp mRNA levels were much lower in Chrebp ${ }^{-/-}$and DKO mice than in WT mice; however, those in Shp ${ }^{-/-}$ mice were only slightly (only 1.15 times) higher than in WT mice (Figure 3A). Consistent with mRNA levels, MTTP protein levels were significantly much lower in Chrebp ${ }^{-/-}$and DKO mice than in WT mice (Figure 3B). Moreover, VLDL TG contents of $\mathrm{Chrebp}^{-/}$and DKO mice were lower than those in WT and Shp ${ }^{-/-}$mice, while VLDL secretion rates in $\mathrm{Chrebp}^{-/-}$and DKO mice were approximately 0.6 times lower than in WT mice (Figure 3C). In contrast, liver VLDL secretion rates in Shp ${ }^{-/}$mice were similar to those in WT mice (Figure 3C). In support of this, the VLDL TG contents and VLDL particle numbers in $\mathrm{Chrebp}^{-1-}$ mice and DKO mice were much lower those in WT mice, while those in Shp ${ }^{-1-}$ mice were similar to those in WT mice (Figure 3D,E). Therefore, under normal conditions, the effect of ChREBP on Mttp expression was potent but the effect of SHP was physiologically much weaker or lacking. 
(A)

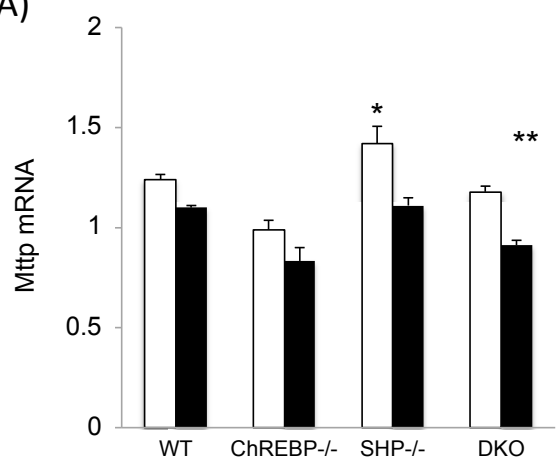

(B)

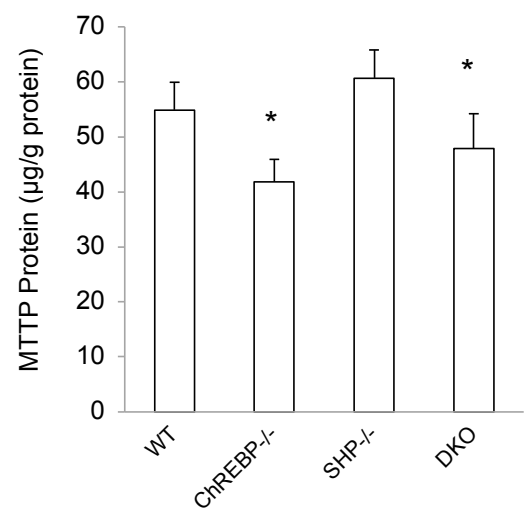

(E)
(C)

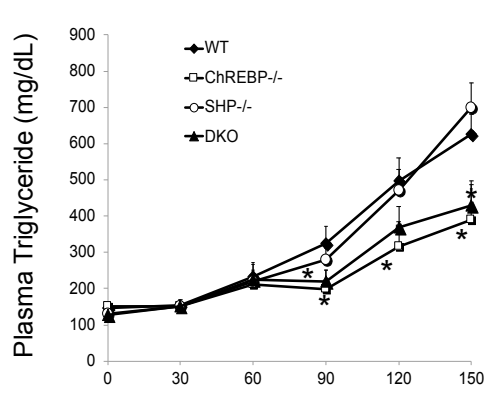

(D)

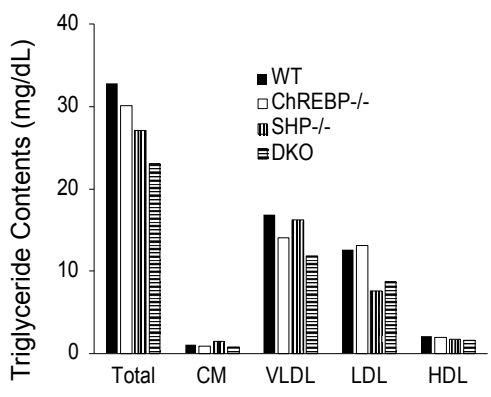

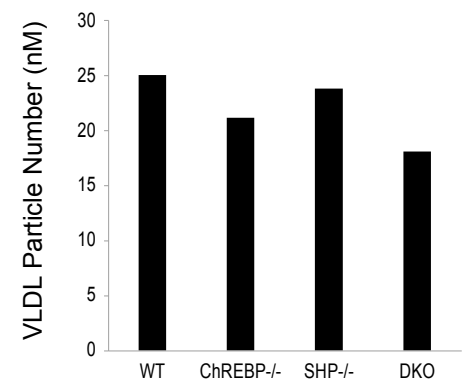

Figure 3. Shp deletion failed to recover decreased Mttp expression, and thereby, VLDL secretion rates mediated by Chrebp deletion. (A) Hepatic Mttp mRNA levels in the livers of wild-type (WT), $\mathrm{Chrebp}^{-/-}, \mathrm{Shp}^{-/-}$, and DKO mice. Mttp mRNA levels were detected by real-time PCR. Pol2 expression was used as an internal control. ${ }^{*} p<0.05$ vs. WT (fast), $n=4 .{ }^{* *} p<0.05$ vs. WT (fed), $n=4$. Data are represented as means \pm SD; (B) Hepatic MTTP protein expression in the livers of WT, $\mathrm{Chrebp}^{-/-}, \mathrm{Shp}^{-/-}$, and DKO mice. MTTP protein levels were measured by ELISA and corrected for total protein levels. ${ }^{*} p<0.05$ vs. WT, $n=5-7$. Data are represented as means \pm SD; (C) VLDL secretion rates were measured as previously reported [17]. ${ }^{*} p<0.05$ vs. WT, $n=5-7$. Data are represented as means \pm SD; TG contents in lipoprotein fraction (D) and VLDL particle numbers (E) were analyzed using gel-permeation high-performance liquid chromatography. Data are representative of six samples pooled.

\section{Discussion}

In this study, we evaluated whether ChREBP and SHP could coordinately affect VLDL secretion via Mttp expression. ChREBP and SHP reciprocally affected Mttp mRNA levels; however, the potency of Mttp suppression by SHP was much lower than that of Mttp induction by ChREBP. Therefore, ChREBP and SHP did not affect the transcriptional activity of each other. Mttp mRNA and protein levels in Chrebp $\mathrm{p}^{-/-}$and DKO mice were much lower than those in WT mice; however, those in Shp $\mathrm{p}^{-/-}$ mice were similar to WT. In agreement with this, VLDL secretion rates of DKO and Chrebp ${ }^{-1-}$ mice were much lower than those of Shp ${ }^{-/-}$mice, which were the same as those of WT mice. Together, these findings suggest that ChREBP, rather than SHP, regulates Mttp expression under normal conditions.

SHP predominantly functions as a transcriptional repressor of gene expression that binds directly to nuclear receptors, such as LRH-1, HNF $4 \alpha$, estrogen receptors, estrogen receptor-related receptors, liver $\mathrm{X}$ receptors, peroxisome proliferator-activated receptors, glucocorticoid receptor, thyroid hormone receptor $\beta$, retinoic acid receptor $\alpha, F X R$, pregnane $X$ receptor, constitutive androstane receptor, androgen receptor, nerve growth factor IB, and common heterodimerization partner retinoid $X$ receptors [10]. SHP was previously shown to suppress MTTP expression by binding to HNF4 $\alpha /$ LRH-1 sites in the Mttp promoter $[10,13,35]$. 
Recent reports have proposed a new ChREBP regulatory mechanism (ChREBP-nuclear receptor interaction), in which nuclear factors interact with ChREBP to modify ChREBP transcriptional activity [25-28]. Computer analysis previously revealed that ChREBP contains an LxQLLT sequence that matches the NR binding motif [29]. The rat LxQLLT sequence is localized to a proline-rich region (540-545 a.a.) of ChREBP [29]. SHP binds and represses the transcriptional activities of target genes by utilizing two functional LXXLL-related motifs located in the ligand-binding domain [10]. Some studies have also suggested that the interactions between ChREBP and nuclear receptors, such as FXR and HNF4, play physiological roles in regulating Pklr expression [25-28]. HNF4 and FXR positively and negatively regulate ChREBP transcriptional activity, respectively. However, our data revealed that SHP overexpression did not affect Pklr mRNA or luciferase activity of the PGL3 3xPKLR ChoRE vector. Moreover, SHP deletion did not affect the mRNA levels of ChREBP target genes ( $P k l r$ and Fgf21) in mice. Consistent with this, we found that ChREBP interacted with SHP in the mammalian two-hybrid system, and interactions with SHP did not interfere with the binding between FXR and ChREBP in the mammalian two-hybrid system (data not shown). This indicated that SHP might not affect the interplay between ChREBP and nuclear factors such as FXR and HNF4, and therefore, that it does not modulate ChREBP transcriptional activity.

Reporter assays for the Mttp promoter $(-211 /+81 \mathrm{bp})$ in the present study revealed that SHP suppressed hepatic Mttp expression, but that ChREBP overexpression did not affect luciferase activity controlled by the Mttp promoter $(-211 /+81 \mathrm{bp})$. This supports the notion that ChREBP does not affect the role of SHP as a corepressor. Taken together with the fact that a Chrebp deletion did not affect SHP target gene (Cyp7a1) mRNA levels, this suggested that ChREBP does not modulate SHP transcriptional activity. Thus, ChREBP and SHP do not affect the transcriptional activities of each other.

To evaluate ChREBP binding to Mttp promoter regions, we searched for putative ChoRE motifs [36-38] in the promoter, exons, and introns. However, we did not detect ChoREs in Mttp. Considering that ChREBP regulates the expression of many genes [21,30,32,33,36-39], we propose that it might indirectly induce Mttp expression through the activation of other transcription factors. However, further investigation is needed to identify the mechanism underlying the ChREBP induction of Mttp expression.

The VLDL secretory pathway is regulated by MTTP, a rate-limiting enzyme. We previously reported that ChREBP positively regulates VLDL secretion [19]. Although SHP controls Mttp expression by modulating HNF4 and LRH-1 transcriptional activity, its effect on VLDL secretion was controversial $[15,16]$. Some studies reported that VLDL secretion in Shp ${ }^{-/-}$mice on ob/ob or C57BL/ 6 backgrounds increased relative to WT mice [16], while others showed that VLDL secretion in $\mathrm{Shp}^{-/-}$mice fed a Western diet was similar to that in $\mathrm{Shp}^{-/-}$mice fed a normal diet [15]. Our data were consistent with the latter. They also confirmed that ChREBP regulates VLDL secretion, but that SHP deletion did not affect VLDL secretion in either WT or Chrebp ${ }^{-1-}$ mice. Our analyses of MTTP protein levels and VLDL particle numbers also supported these data. Taken together with the fact that SHP overexpression by more than 70 times was needed to suppress ChREBP-mediated Mttp induction, these findings suggest that ChREBP plays important roles in VLDL secretion, while those of SHP are much smaller or negligible.

Nutritional conditions affect the development of NAFLD and dyslipidemia. High carbohydrate diets and high fat diets increase Mttp expression [9]. High carbohydrate diets increase de novo lipogenesis and Mttp expression [9,18]. As we previously reported, Chrebp deletions prevent hepatic lipid accumulation following consumption of a high carbohydrate diet $[30,40]$. The prevention from high carbohydrate diet induced fatty liver was due to a decrease in de novo lipogenesis, decreased free fatty acid supply from adipose tissue, and appetite loss from the high sucrose and high fructose diet [30,40-43]. Decreased Mttp expression might deteriorate NAFLD in ChREBP ${ }^{-/-}$mice. As ChREBP ${ }^{-/-}$mice could not be fed a high carbohydrate diet such as the high sucrose diet [30,41,42], we could not perform an experiment using the high carbohydrate diet. In contrast, a high fat diet increased the FFA supply from dietary fat and induced Mttp expression [17]. The High fat diet 
suppressed ChREBP transcriptional activity [44]. Moreover, Chrebp deletion did not prevent from high fat diet induced fatty liver owing to decreased fatty acid oxidation [19,43]. As Shp suppress PPAR alpha [45], Shp deletion prevented hepatic lipid accumulation through increased fatty acid oxidation [15]. Consistent with our data, Mttp expression was not affected by Shp deletion. Therefore, we analyzed the effects of ChREBP and SHP deletion on Mttp expression and VLDL secretion under a normal diet condition. In this study, ChREBP and SHP independently regulated glucose and lipid metabolism. The difference in target genes between ChREBP and SHP might contribute to the different effects of ChREBP and SHP on preventing the development of fatty liver induced by high fat diets in Chrebp $^{-/-}$and Shp ${ }^{-/-}$mice (Figure 4 ).

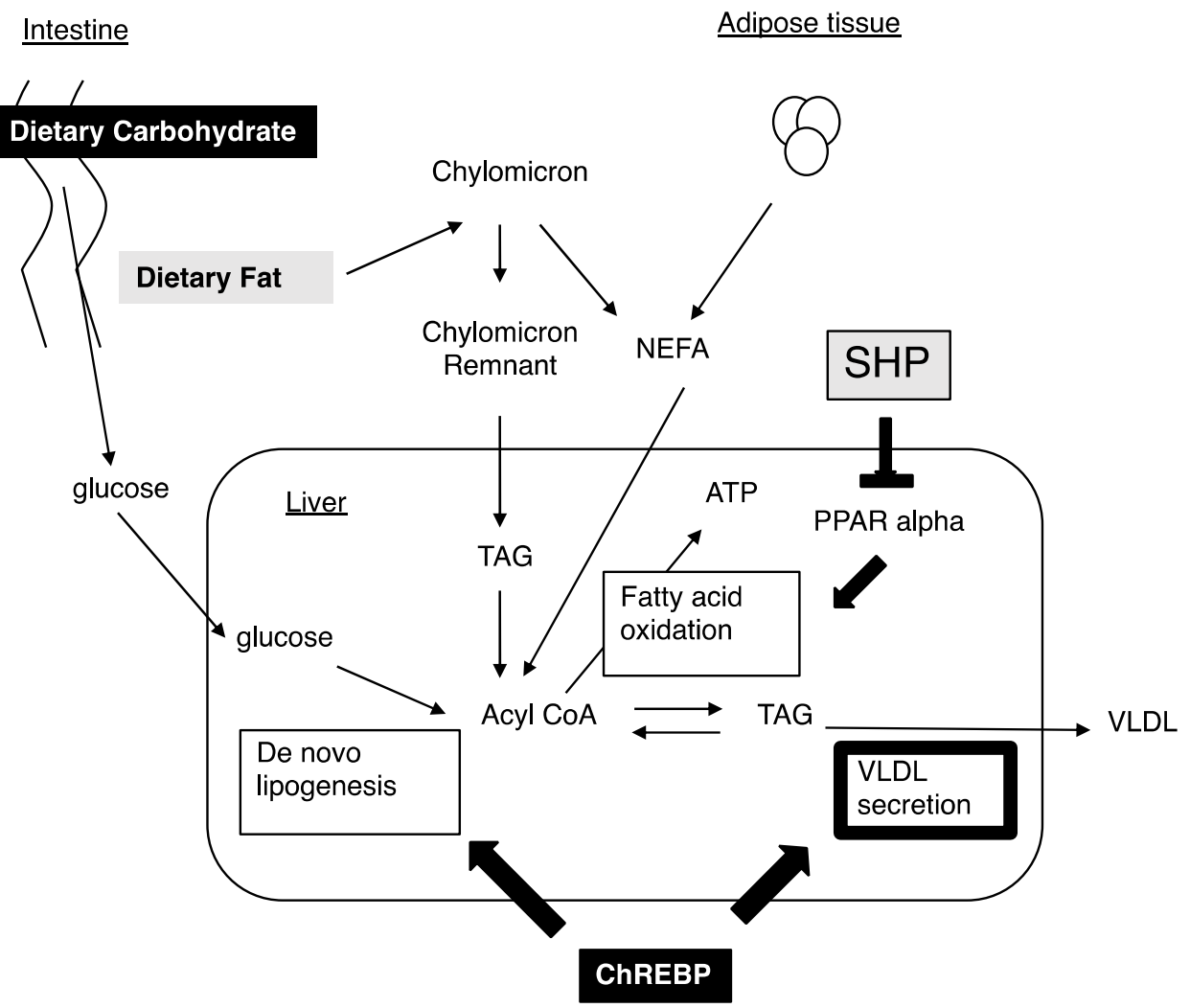

Figure 4. ChREBP and SHP independently regulate hepatic lipid metabolism. High carbohydrate diets increase de novo lipogenesis and VLDL secretion through ChREBP activation. Therefore, Chrebp deletion prevents high carbohydrate diet induced fatty liver [30,40,42,43]. In contrast, SHP suppresses PPAR alpha and an Shp deletion prevents high fat diet induced fatty liver [15,45]. In this study, we clarified that ChREBP, rather than SHP, regulates VLDL secretion through Mttp expression (bold line). Thus, ChREBP and SHP independently regulate hepatic lipid metabolism.

\section{Conclusions}

ChREBP and SHP were shown to modulate hepatic Mttp expression, but the capacity of Mttp suppression by SHP was much lower than that of Mttp induction by ChREBP. Moreover, ChREBP and SHP did not affect the transcriptional activities of each other. Unlike many transcription factors, ChREBP was not regulated by SHP. Finally, ChREBP, rather than SHP, was shown to regulate hepatic VLDL secretion.

Acknowledgments: We thank Sarah Williams, from Edanz Group (www.edanzediting.com) for editing a draft of this manuscript. This work was supported in part by a Grant-in-Aid for Scientific Research from the Japan Society for the Promotion of Science (Iizuka, K.: No. 17K00850, 26500005, Takeda, J.: No. 17K19902), research grants from MSD and Novartis Pharma. (Iizuka, K. and Takeda, J.). 
Author Contributions: K.I. conceived and designed the experiments; H.N., T.K., W.W., H.T. and K.T. performed the experiments; H.N. analyzed the data; Y.H. gave a support in the literature review; K.I. and J.T. wrote and revised the paper. All the authors approved the final version of the manuscript.

Conflicts of Interest: The authors declare no conflicts of interest.

\section{References}

1. Fabbrini, E.; Magkos, F. Hepatic Steatosis as a Marker of Metabolic Dysfunction. Nutrients 2015, 7, 4995-5019. [CrossRef] [PubMed]

2. Fabbrini, E.; Sullivan, S.; Klein, S. Obesity and nonalcoholic fatty liver disease: Biochemical, metabolic, and clinical implications. Hepatology 2010, 51, 679-689. [CrossRef] [PubMed]

3. Agius, L. High-carbohydrate diets induce hepatic insulin resistance to protect the liver from substrate overload. Biochem. Pharmacol. 2013, 85, 306-312. [CrossRef] [PubMed]

4. Westerbacka, J.; Lammi, K.; Häkkinen, A.M.; Rissanen, A.; Salminen, I.; Aro, A.; Yki-Järvinen, H. Dietary fat content modifies liver fat in overweight nondiabetic subjects. J. Clin. Endocrinol. Metab. 2005, 90, 2804-2809. [CrossRef] [PubMed]

5. Yu, J.; Marsh, S.; Hu, J.; Feng, W.; Wu, C. The Pathogenesis of Nonalcoholic Fatty Liver Disease: Interplay between Diet, Gut Microbiota, and Genetic Background. Gastroenterol. Res. Pract. 2016, 2016, 2862173. [CrossRef] [PubMed]

6. Donnelly, K.L.; Smith, C.I.; Schwarzenberg, S.J.; Jessurun, J.; Boldt, M.D.; Parks, E.J. Sources of fatty acids stored in liver and secreted via lipoproteins in patients with nonalcoholic fatty liver disease. J. Clin. Investig. 2005, 115, 1343-1351. [CrossRef] [PubMed]

7. Browning, J.D.; Horton, J.D. Molecular mediators of hepatic steatosis and liver injury. J. Clin. Investig. 2004, 114, 147-152. [CrossRef] [PubMed]

8. Cuchel, M.; Rader, D.J. Microsomal transfer protein inhibition in humans. Curr. Opin. Lipidol. 2013, 24, 246-250. [CrossRef] [PubMed]

9. Hussain, M.M.; Nijstad, N.; Franceschini, L. Regulation of microsomal triglyceride transfer protein. Clin. Lipidol. 2011, 6, 293-303. [CrossRef] [PubMed]

10. Zhang, Y.; Hagedorn, C.H.; Wang, L. Role of nuclear receptor SHP in metabolism and cancer. Biochim. Biophys. Acta 2011, 1812, 893-908. [CrossRef] [PubMed]

11. Nishigori, H.; Tomura, H.; Tonooka, N.; Kanamori, M.; Yamada, S.; Sho, K.; Inoue, I.; Kikuchi, N.; Onigata, K.; Kojima, I.; et al. Mutations in the small heterodimer partner gene are associated with mild obesity in Japanese subjects. Proc. Natl. Acad. Sci. USA 2001, 98, 575-580. [CrossRef] [PubMed]

12. Enya, M.; Horikawa, Y.; Kuroda, E.; Yonemaru, K.; Tonooka, N.; Tomura, H.; Oda, N.; Yokoi, N.; Yamagata, K.; Shihara, N.; et al. Mutations in the small heterodimer partner gene increase morbidity risk in Japanese type 2 diabetes patients. Hum. Mutat. 2008, 29, E271-E277. [CrossRef] [PubMed]

13. Lee, Y.K.; Dell, H.; Dowhan, D.H.; Hadzopoulou-Cladaras, M.; Moore, D.D. The orphan nuclear receptor SHP inhibits hepatocyte nuclear factor 4 and retinoid $X$ receptor transactivation: Two mechanisms for repression. Mol. Cell. Biol. 2000, 20, 187-195. [CrossRef] [PubMed]

14. Goodwin, B.; Jones, S.A.; Price, R.R.; Watson, M.A.; McKee, D.D.; Moore, L.B.; Galardi, C.; Wilson, J.G.; Lewis, M.C.; Roth, M.E.; et al. A regulatory cascade of the nuclear receptors FXR, SHP-1, and LRH-1 represses bile acid biosynthesis. Mol. Cell 2000, 6, 517-526. [CrossRef]

15. Park, Y.J.; Kim, S.C.; Kim, J.; Anakk, S.; Lee, J.M.; Tseng, H.T.; Yechoor, V.; Park, J.; Choi, J.S.; Jang, H.C.; et al. Dissociation of diabetes and obesity in mice lacking orphan nuclear receptor small heterodimer partner. J. Lipid Res. 2011, 52, 2234-2244. [CrossRef] [PubMed]

16. Huang, J.; Iqbal, J.; Saha, P.K.; Liu, J.; Chan, L.; Hussain, M.M.; Moore, D.D.; Wang, L. Molecular characterization of the role of orphan receptor small heterodimerpartner in development of fatty liver. Hepatology 2007, 46, 147-157. [CrossRef] [PubMed]

17. Lin, M.C.; Arbeeny, C.; Bergquist, K.; Kienzle, B.; Gordon, D.A.; Wetterau, J.R. Cloning and regulation of hamster microsomal triglyceride transfer protein. The regulation is independent from that of other hepatic and intestinal proteins which participate in the transport of fatty acids and triglycerides. J. Biol. Chem. 1994, 269, 29138-29145. [PubMed] 
18. Mock, K.; Lateef, S.; Benedito, V.A.; Tou, J.C. High-fructose corn syrup-55 consumption alters hepatic lipid metabolism and promotes triglyceride accumulation. J. Nutr. Biochem. 2017, 39, 32-39. [CrossRef] [PubMed]

19. Wu, W.; Tsuchida, H.; Kato, T.; Niwa, H.; Horikawa, Y.; Takeda, J.; Iizuka, K. Fat and carbohydrate in western diet contribute differently to hepatic lipid accumulation. Biochem. Biophys. Res. Commun. 2015, 461, 681-686. [CrossRef] [PubMed]

20. Uyeda, K.; Repa, J.J. Carbohydrate response element binding protein, ChREBP, a transcription factor coupling hepatic glucose utilization and lipid synthesis. Cell Metab. 2006, 4, 107-110. [CrossRef] [PubMed]

21. Iizuka, K. The transcription factor carbohydrate-response element-binding protein (ChREBP): A possible link between metabolic disease and cancer. Biochim. Biophys. Acta 2017, 1863, 474-485. [CrossRef] [PubMed]

22. Abdul-Wahed, A.; Guilmeau, S.; Postic, C. Sweet Sixteenth for ChREBP: Established Roles and Future Goals. Cell Metab. 2017, 26, 324-341. [CrossRef] [PubMed]

23. Herman, M.A.; Samuel, V.T. The Sweet Path to Metabolic Demise: Fructose and Lipid Synthesis. Trends Endocrinol. Metab. 2016, 27, 719-730. [CrossRef] [PubMed]

24. Poupeau, A.; Postic, C. Cross-regulation of hepatic glucose metabolism via ChREBP and nuclear receptors. Biochim. Biophys. Acta 2011, 1812, 995-1006. [CrossRef] [PubMed]

25. Caron, S.; Huaman Samanez, C.; Dehondt, H.; Ploton, M.; Briand, O.; Lien, F.; Dorchies, E.; Dumont, J.; Postic, C.; Cariou, B.; et al. Farnesoid X receptor inhibits the transcriptional activity of carbohydrate response element binding protein in human hepatocytes. Mol. Cell. Biol. 2013, 33, 2202-2211. [CrossRef] [PubMed]

26. Meng, J.; Feng, M.; Dong, W.; Zhu, Y.; Li, Y.; Zhang, P.; Wu, L.; Li, M.; Lu, Y.; Chen, H.; et al. Identification of HNF- $4 \alpha$ as a key transcription factor to promote ChREBP expression in response to glucose. Sci. Rep. 2016, 6, 23944. [CrossRef] [PubMed]

27. Trabelsi, M.S.; Daoudi, M.; Prawitt, J.; Ducastel, S.; Touche, V.; Sayin, S.I.; Perino, A.; Brighton, C.A.; Sebti, Y.; Kluza, J.; et al. Farnesoid $X$ receptor inhibits glucagon-like peptide-1 production by enteroendocrine $\mathrm{L}$ cells. Nat. Commun. 2015, 6, 7629. [CrossRef] [PubMed]

28. Burke, S.J.; Collier, J.J.; Scott, D.K. cAMP opposes the glucose-mediated induction of the L-PK gene by preventing the recruitment of a complex containing ChREBP, HNF4alpha, and CBP. FASEB J. 2009, 23, 2855-2865. [CrossRef] [PubMed]

29. McFerrin, L.G.; Atchley, W.R. A novel N-terminal domain may dictate the glucose response of Mondo proteins. PLoS ONE 2012, 7, e34803. [CrossRef] [PubMed]

30. Iizuka, K.; Bruick, R.K.; Liang, G.; Horton, J.D.; Uyeda, K. Deficiency of carbohydrate response element-binding protein (ChREBP) reduces lipogenesis as well as glycolysis. Proc. Natl. Acad. Sci. USA 2004, 101, 7281-7286. [CrossRef] [PubMed]

31. Bligh, E.G.; Dyer, W.J. A rapid method of total lipid extraction and purification. Can. J. Biochem. Physiol. 1959, 37, 911-917. [CrossRef] [PubMed]

32. Iizuka, K.; Horikawa, Y. Regulation of lipogenesis via BHLHB2/DEC1 and ChREBP feedback looping. Biochem. Biophys. Res. Commun. 2008, 374, 95-100. [CrossRef] [PubMed]

33. Iizuka, K.; Takeda, J.; Horikawa, Y. Glucose induces FGF21 mRNA expression through ChREBP activation in rat hepatocytes. FEBS Lett. 2009, 583, 2882-2886. [CrossRef] [PubMed]

34. Okazaki, M.; Yamashita, S. Recent Advances in Analytical Methods on Lipoprotein Subclasses: Caluculation of Particle Numbers from Lipid Levels by Gel Permeation HPLC Using "Spherical Particle Model”. J. Oleo Sci. 2016, 65, 265-282. [CrossRef] [PubMed]

35. Hirokane, H.; Nakahara, M.; Tachibana, S.; Shimizu, M.; Sato, R. Bile acid reduces the secretion of very low density lipoprotein by repressing microsomal triglyceridetransfer protein gene expression mediated by hepatocyte nuclear factor-4. J. Biol. Chem. 2004, 279, 45685-45692. [CrossRef] [PubMed]

36. Jeong, Y.S.; Kim, D.; Lee, Y.S.; Kim, H.J.; Han, J.Y.; Im, S.S.; Chong, H.K.; Kwon, J.K.; Cho, Y.H.; Kim, W.K.; et al. Integrated expression profiling and genome-wide analysis of ChREBP targets reveals the dual role for ChREBP in glucose-regulated gene expression. PLoS ONE 2011, 6, e22544. [CrossRef] [PubMed]

37. Poungvarin, N.; Chang, B.; Imamura, M.; Chen, J.; Moolsuwan, K.; Sae-Lee, C.; Li, W.; Chan, L. Genome-Wide Analysis of ChREBP Binding Sites on Male Mouse Liver and White Adipose Chromatin. Endocrinology 2015, 156, 1982-1994. [CrossRef] [PubMed]

38. Ma, L.; Robinson, L.N.; Towle, H.C. ChREBP*Mlx is the principal mediator of glucose-induced gene expression in the liver. J. Biol. Chem. 2006, 281, 28721-28730. [CrossRef] [PubMed] 
39. Iizuka, K. The Role of Carbohydrate Response Element Binding Protein in Intestinal and Hepatic Fructose Metabolism. Nutrients 2017, 9, 181. [CrossRef] [PubMed]

40. Iizuka, K.; Miller, B.; Uyeda, K. Deficiency of carbohydrate-activated transcription factor ChREBP prevents obesity and improves plasma glucose control in leptin-deficient (ob/ob) mice. Am. J. Physiol. Endocrinol. Metab. 2006, 291, E358-E364. [CrossRef] [PubMed]

41. Kim, M.; Astapova, I.I.; Flier, S.N.; Hannou, S.A.; Doridot, L.; Sargsyan, A.; Kou, H.H.; Fowler, A.J.; Liang, G.; Herman, M.A. Intestinal, but not hepatic, ChREBP is required for fructose tolerance. JCI Insight 2017, 2 , e96703. [CrossRef] [PubMed]

42. Linden, A.G.; Li, S.; Choi, H.Y.; Fang, F.; Fukasawa, M.; Uyeda, K.; Hammer, R.E.; Horton, J.D.; Engelking, L.J.; Liang, G. Interplay between ChREBP and SREBP-1c Coordinates Postprandial Glycolysis and Lipogenesis in Livers of Mice. J. Lipid Res. 2018, 59, 475-487. [CrossRef] [PubMed]

43. Burgess, S.C.; Iizuka, K.; Jeoung, N.H.; Harris, R.A.; Kashiwaya, Y.; Veech, R.L.; Kitazume, T.; Uyeda, K. Carbohydrate-response element-binding protein deletion alters substrate utilization producing an energy-deficient liver. J. Biol. Chem. 2008, 283, 1670-1678. [CrossRef] [PubMed]

44. Kim, J.K.; Lee, K.S.; Chang, H.Y.; Lee, W.K.; Lee, J.I. Progression of diet induced nonalcoholic steatohepatitis is accompanied by increased expression of kruppel-like-factor 10 in mice. J. Transl. Med. 2014, 12, 186. [CrossRef] [PubMed]

45. Kassam, A.; Capone, J.P.; Rachubinski, R.A. The short heterodimer partner receptor differentially modulates peroxisome proliferator-activated receptor alpha-mediated transcription from the peroxisome proliferator-response elements of the genes encoding the peroxisomal beta-oxidation enzymes acyl-CoA oxidase and hydratase-dehydrogenase. Mol. Cell. Endocrinol. 2001, 176, 49-56. [PubMed]

(C) 2018 by the authors. Licensee MDPI, Basel, Switzerland. This article is an open access article distributed under the terms and conditions of the Creative Commons Attribution (CC BY) license (http:/ / creativecommons.org/licenses/by/4.0/). 